\title{
Variations chétotaxiques des cercaires d'une souche humaine de Scbistosoma mansoni par passages successifs sur Souris
}

\author{
par J. CASSONE *, Ch. BAYSSADE-DUFOUR *, J.-L. ALBARET * \\ et J. JOURDANE ** \\ (Collaboration technique: B. LABRousSE **) \\ * Laboratoire de Zoologie (Vers) associé au C.N.R.S. \\ Museum national d'Histoire naturelle, 43, rue Cuvier, F 75231 Paris Cedex 05. \\ * Département de Biologie animale, Centre Universitaire, \\ F 66025 Perpignan Cedex
}

\section{Résuméé}

L'indice chétotaxique cercarien d'une souche humaine de Schistosoma mansoni d'Afrique Occidentale ne se modifie nettement qu'au troisième passage par Biomphalaria pfeifferi, souris blanche.

Cela pourrait expliquer pourquoi les Planorbes récoltées en Guadeloupe émettent des cercaires peu différentes, qu'elles proviennent de gîtes où l'infestation est à prédominance humaine ou de gîtes où l'infestation est à prédominance murine. La station de « Grand Etang 》, au contraire, s'oppose significativement à toutes les autres et paraît à transmission exclusivement murine.

\section{Summary.}

Chaetotaxic variations of the cercaria Schistosoma mansoni from a human strain during successive transmissions through mice.

The "cercarian chaetotaxic index » from the human strain of Schistosoma mansoni from West Africa is conspicuously modified during the third transmission Biomphalaria pfeifferi white mouse only.

Accepté le 13 février 1978. 
This might be the explanation why Planorbs collected in Guadeloupe discharge slightly different cercariae whether they come from stations where the infestation is predominantly from human origin or from stations where the infestation is predominantly from murine origin. On the contrary, the "Grand Etang 》 station is significatively different from all the others and seems to be exclusively murine transmitted.

Lors d'une précédente étude sur les variations chétotaxiques des cercaires de Schistosoma mansoni, l'un de nous (Bayssade-Dufour, 1977) a mis en évidence une différence caractéristique entre les souches humaines et murines.

Si $x_{1}$ est la distance entre les papilles AIL et AIIL et $y_{1}$ la distance entre les papilles AID et AIID, on définit un indice par hémicorps: $n_{1}=\frac{x_{1}}{y_{1}}$ et $n_{2}=\frac{x_{2}}{y_{2}}$, ce qui donne pour chaque cercaire un indice $n=\frac{1}{2}\left(n_{1}+n_{2}\right)$. Une moyenne arithmétique de l'indice est ainsi obtenue pour chaque souche, ainsi que l'intervalle de confiance avec une sécurité de $95 \%$. Cet indice est sensiblement égal à 1 pour les souches humaines d'Afrique occidentale, entre 1,1 et 1,3 pour les souches humaines d'Amérique, de 1,7 pour une souche entretenue en laboratoire depuis très longtemps sur souris et de 1,6 pour une souche passant par rat sauvage en Guadeloupe.

Nous avons été amenés à chercher comment une souche humaine pouvait acquérir l'indice murin, puisqu'après un seul passage expérimental sur rat ou souris, l'indice ne change pas, alors qu'après de nombreuses générations, il est de 1,77. Disposant d'une souche humaine d'Afrique occidentale (souche «Père blanc », Guinée ou R.C.A., déjà utilisée par Bayssade-Dufour, 1977), nous lui avons fait subir trois passages successifs sur Biomphalaria pfeifferi et souris blanche.

\section{Résultats}

Les résultats bruts (1) de ces expériences sont donnés dans les histogrammes A. B. C. D de la figure I; chacun d'eux (A, B et C) est obtenu à partir de l'étude de 60 cercaires. Pour D, l'étude a été faite sur 120 cercaires et les effectifs divisés par 2 pour rendre la comparaison graphique plus aisée avec les 3 précédents. Pour chacune de ces populations, on a défini la moyenne arithmétique des indices, la variance $\left(\sigma^{2}\right)$ et l'intervalle de confiance $(2 s)$, récapitulés dans le tableau suivant

(1) Les différentes données numériques sont déposées dans les collections du Muséum National d'Histoire Naturelle (Laboratoire de Zologie - Vers) et peuvent être communiquées sur demande.

41 cercaires ne figurent pas dans les histogrammes pour les raisons suivantes: Papilles en surnombre (4 chez A, 2 chez C). Une des quatre papilles absente ( 8 chez A, 5 chez B, 1 chez C, 13 chez D). Aberrations diverses (5 chez A, 3 chez D). 

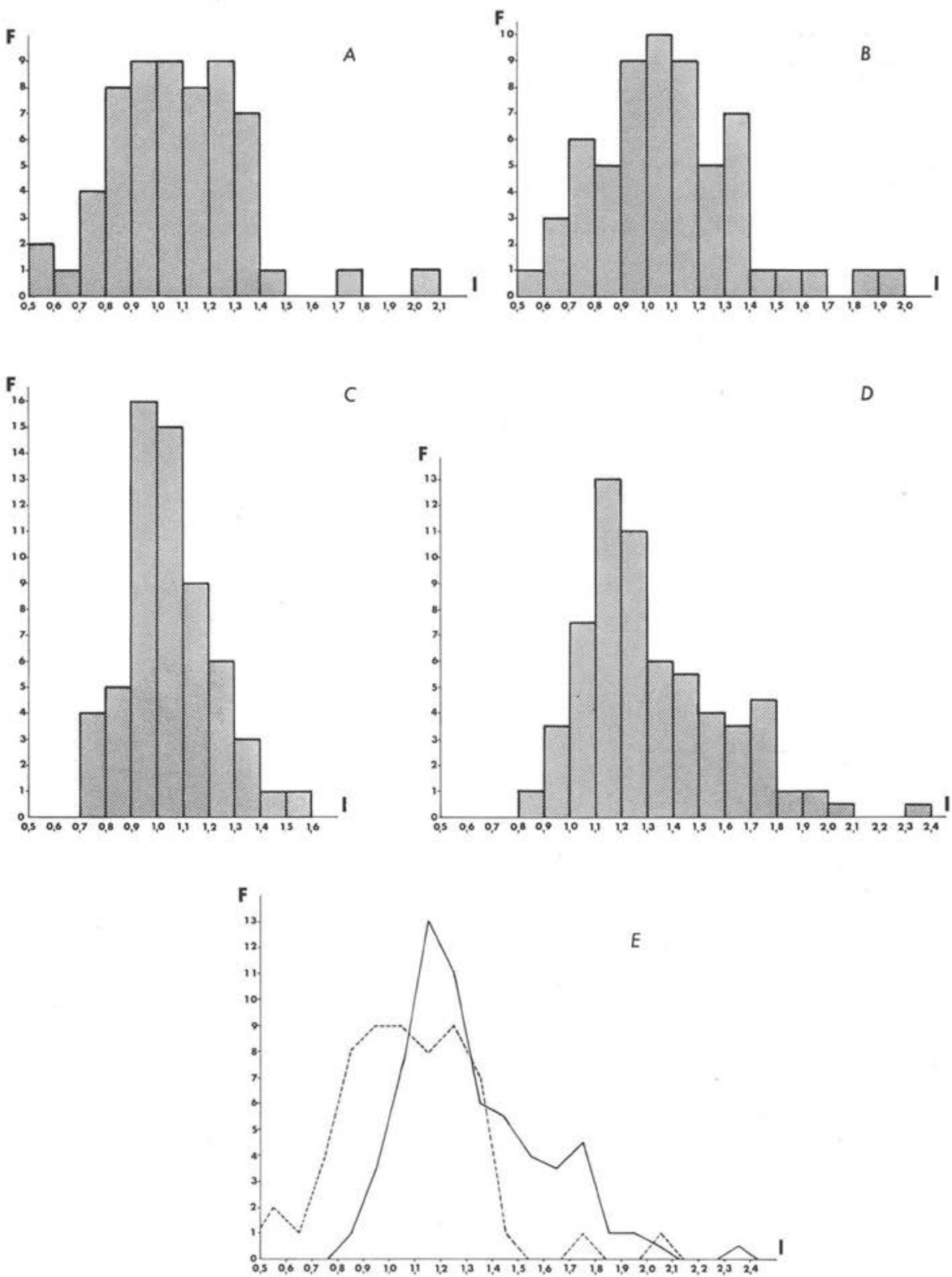

Fig. 1. Histogramme de répartition des populations de cercaires en fonction de l'indice et de la fréquence de classe. A. - souche humaine. B - C - D, respectivement $1^{\mathrm{er}}, 2^{\mathrm{e}}, 3^{\mathrm{e}}$, passage sur Souris. E. - comparaison des distributions A (en pointillés) et D (trait plein). 


\begin{tabular}{|c|c|c|c|c|}
\hline & A & B & C & D \\
\hline ………… & 1,08 & 1,07 & 1,05 & 1,30 \\
\hline$\sigma^{2} \ldots \ldots \ldots \ldots \ldots$ & 0,060 & 0,072 & 0,024 & 0,058 \\
\hline $2 \mathrm{~s} \ldots \ldots \ldots \ldots$ & 0,06 & 0,06 & 0,04 & 0,04 \\
\hline $\mathrm{N}, \ldots \ldots \ldots \ldots, \ldots$ & 60 & 60 & 60 & 123 \\
\hline
\end{tabular}

Un test d'ajustement à une loi normale montre que les populations $\mathrm{A}, \mathrm{B}, \mathrm{C}$ ont une distribution Gaussienne avec un $\%^{2}$ dont la valeur est largement en-deça de la limite de probabilité d'erreur de $5 \%$. En revanche, l'hypothèse d'une distribution gaussienne pour la $4^{\mathrm{e}}$ courbe est à rejeter : le $\%^{2}$ est largement au-delà de la limite d'erreur de $5 \%$ fixée. Ceci montre que cette dernière population est significativement différente des trois autres dont elle est issue.

En appliquant un test de comparaison de variance (test $\mathrm{F}$ ) aux trois premières populations, on montre que $\mathrm{A}$ et $\mathrm{B}$ ne diffèrent pas significativement, alors que $\mathrm{C}$ diffère significativement des deux autres. On remarque, en effet, sur cette dernière population, un resserrement de la base, avec élimination (du moins dans l'effectif de population envisagé) des extrêmes.

\section{Discussion}

L'analyse des courbes obtenues indique que la dispersion des mesures est moins importante lors du $2^{\circ}$ passage, ce qui paraît correspondre à une sélection. Au troisième passage, au contraire, la distribution est asymétrique, avec augmentation du nombre d'individus d'indice fort. Le fait qu'elle ne soit pas ajustable à une loi normale n'est pas incompatible avec l'hypothèse d'un mélange de deux populations distinctes, une ayant une moyenne correspondant à la souche humaine $(1,1)$ et une seconde avec la moyenne de 1,7 correspondant à celle des souches entretenues depuis de nombreuses générations sur la souris.

Cette modification au $3^{e}$ passage seulement s'explique assez difficilement. Nous notons cependant que les expérimentateurs (1) s'accordent à constater qu'une souche humaine s'adapte aisément au cours du premier et du deuxième passage aux conditions expérimentales: plus de $50 \%$ des mollusques s'infestent aisément, alors que lors des $3^{\circ}$ et $4^{\circ}$ passages, la plupart des mollusques exposés aux miracidiums issus de la $2^{\circ}$ et $3^{\circ}$ génération restent négatifs et il est fréquent de perdre la souche à ce moment.

Dans un troisième temps, au contraire, lors du $5^{\mathrm{e}}$ et $6^{\mathrm{e}}$ passage, la souche paraît s'adapter à la souris et les passages successifs redeviennent aisés. Il semble donc y avoir une corrélation entre la modification biologique de la souche et la modification de l'indice chétotaxique des cercaires.

(1) Nous remercions nos collègues $\mathrm{Cl}$. Combes, R. Houin, J. Lapierre et $\mathrm{H}$. Picot de nous avoir fait part de leurs remarques sur ce point. 


\section{Données épidémiologiques}

En Guadeloupe, dans des biotopes où la bilharziose paraît typiquement humaine (cf. Rioux et coll.), l'indice chétotaxique de 52 Mollusques a été déterminé. Il varie de 1,05 à 1,50 pour 49 Mollusques. 3 seulement ont un indice un peu supérieur : $1,57( \pm 0,20), 1,58( \pm 0,28)$ et $1,67( \pm 0,34)$. Pour 3 Mollusques récoltés en arrièremangrove, dans un biotope où la bilharziose murine est intense (cf. Mougeot et Golvan, 1977), les indices sont de 1,43 ( $\pm 0,12), 1,37( \pm 0,14)$ et $1,38( \pm 0,06)$.

Ces chiffres ne sont pas comparables à ceux de nos expériences puisque l'indice moyen de l'Homme en Afrique occidentale est d'environ 1,0 et non d'environ 1,3 comme en Amérique.

Cependant, les données expérimentales précédentes montrant que l'indice ne commence à se modifier qu'après le troisième passage sur la Souris, pourraient expliquer pourquoi la «bilharzione murine » de l'arrière mangrove n'a pas un indice très différent de la « bilharziose humaine ». Il y aurait, en périphérie de l'arrière-mangrove, des interférences homme-rat.

A ces résultats s'oppose le gîte de Grand-Etang, où les indices chétotaxiques des souches examinées, qu'elles viennent du Rat ou qu'elles viennent des Mollusques, s'élèvent à $1,63( \pm 0,06)$. Ceci nous paraît un élement objectif qui confirme les conclusions de Combes, Léger et Golvan (1975) sur la nature exclusivement murine du gîte de Grand-Etang.

\section{Bibliographie}

Bayssade-Dufour Ch. (1977): Variation chétotaxique chez les cercaires de Schistosoma mansoni, agent de la bilharziose intestinale, en corrélation avec l'hôte Vertébré de ce parasite. C.R. Acad. Sci., 285, série D, 1511-1513.

Combes C., Léger N., Golvan Y. J. (1975): Le rôle du rat dans la dynamique de l'endémie schistosomienne en Guadeloupe. C.R. Acad. Sci., 281, série D, 1059-1061.

Mougeot G., Golvan Y.J. (1977): Aspects de la bilharziose murine dans la mangrove de la Grande-Terre en Guadeloupe. Ann. Parasitol. hum. com., 52, 623-628.

Nassi H., Lancastre F., Poirot J.-L. (1975) : Rats et Bilharziose en Guadeloupe. Ann. Parasitol. hum. comp., 50, 731-733.

Rioux J.-A., Décamps H., Combes C., Lanotte G., Théron A., Pointier J.-P., Seytor S., Delattre P., Boujerol C. (1977): La Schistosomose intestinale en Guadeloupe. Note 15, D.G.R.S.T., comité équilibre et lutte biologique, groupe méthodologie, novembre 1977, 29 p. 